\title{
Sensory Modalities and Novel Features of Perceptual Experiences
}

\author{
Douglas C. Wadle
}

Is the flavor of mint reducible to the minty smell, the taste, and the menthol-like coolness on the roof of one's mouth, or does it include something over and above these - something not properly associated with any one of the contributing senses? More generally, are there features of perceptual experiences - so-called novel features - that are not associated with any of the standard Aristotelian senses (vision, audition, touch, taste, and smell) taken singly (the flavor of mint being one of the prime candidates for such features)? This question has received a lot of attention of late. ${ }^{1}$ Yet surprisingly little attention has been paid to the question of what it means to say that a feature is associated with a modality in the first place. ${ }^{2}$ Indeed, there is only one fully developed proposal in the literature (O'Callaghan, 2014a, 2014b, 2015, 2017). I argue that this proposal is too permissive to inform the debate over novel features. I go on to argue that all attempts to formulate a better proposal along these lines fail. The corollary of my arguments is that the question of the existence of novel features is poorly formed. Furthermore, the problem generalizes, with the result we should not rely on our pre-theoretical notions of the senses as the basis of theorizing about the features (contents and phenomenal character) of perceptual experiences.

\footnotetext{
${ }^{1}$ See Bayne (2014), Briscoe (2016a, 2016b, 2019), Connolly (2014), Fulkerson (2014a), Macpherson (2011a), O'Callaghan (2008, 2012, 2014a, 2014b, 2015, 2017a, 2017b, 2019). See Spence and Bayne (2014) for a critical look at the evidence for multisensory experiences (with or without novel features).

${ }^{2} \mathrm{~A}$ bit more, but still too little, attention has been paid to another unresolved problem that undermines the attempt to answer this question - namely, that there is no uncontroversial demarcation of the senses on which to base these associations. While I focus on the problem of feature association, I will engage with the further problem of individuating the senses to the extent necessary to address that first problem (see, especially, section 4.2).
} 


\section{Background and Significance}

The philosophy of perception has come a long way in the last two decades. As recently as 1999 one could be forgiven for confusing the philosophy of perception with the philosophy of vision, but since then great strides have been taken to broaden our perspective, first opening new avenues for philosophical research into the senses of hearing, taste, smell, and touch, and now beginning to transcend the boundaries of these senses themselves. We are now able to ask questions like the one with which I began: Are there distinctively multisensory (i.e., novel) features of perceptual experiences? In what follows I will argue that this progress has not taken us far enough. Multisensory approaches to perception retain the inheritance of the earlier sense-by-sense approach, and this inheritance impedes further progress in the philosophy of perception.

When we take the senses one at a time, the complexities introduced by their interactions aren't salient, and it is easy to think that our intuitive associations of features with these senses are unproblematic (and exhaustive). Unfortunately, this unreflective reliance on intuitive feature associations is still evident in much of the discussion of multisensory experience. Bayne (2014) is representative here. After acknowledging that there is no clear rule for associating a given feature of a perceptual experience with a sense and that there is no general agreement on how to individuate the senses, he remarks, "I will (largely) set [these issues] to one side here. As we will see, significant progress can be made in evaluating the decomposition thesis by relying only on our intuitive sense of how to individuate experiences" (p. 16). (The decomposition thesis is the claim that there are no novel features, and by "individuate experiences" Bayne means carve a perceptual experience up into sense-specific components - i.e., assign features of the experience to one or another of the Aristotelian senses - insofar as is possible.) But it is exactly at the interactions of the senses that the relevant intuitions start to break down. We need some other basis for associating features with the senses to properly undertake the multisensory account of perception. 
O'Callaghan is alone in offering a thoroughgoing feature association rule. ${ }^{3}$ As we shall see, though, his proposal won't actually settle the novel feature question. Furthermore, no proposal will. Indeed, no proposal can even deliver all the uncontroversial associations of features with our intuitive, pre-theoretical understandings of the Aristotelian senses - at least not in a way that is otherwise adequate for settling the novel feature question. The lesson I draw is that our pretheoretical notions of the senses, in terms of which the novel feature debate is couched, are an inadequate basis for theorizing about the content and phenomenal character of perceptual experience. ${ }^{4}$ Though I focus on the novel feature debate, this point holds for any debate concerning the content or phenomenology of perceptual experience (e.g., intentionalism, ${ }^{5}$ the thick/thin contents of experience debate, cognitive penetration, etc.). I recommend that we abandon the last vestiges of the sense-by-sense approach. Instead, we should focus on the contributions of the relevant perceptual mechanisms to perceptual experience, without worrying over how these mechanisms relate to our pre-theoretical notions of the (Aristotelian) senses. (More on mechanisms and senses below.) As the novel feature debate is my point of entry, the first step will be to get a clearer understanding of what these novel features are. It is to this that I now turn.

\section{Novel Features}

\footnotetext{
${ }^{3}$ Others have recently adopted O'Callaghan's proposal (e.g., Briscoe 2019), but none have held it up to scrutiny as I shall do. Fulkerson $(2011,2014)$ proposes his own partial rule based in feature binding. By his own admission, though, binding is "at best a sufficient condition" for features to be associated with a given modality (2014b, p. 34). Adjudicating the novel feature debate requires a complete remedy. So I will set Fulkerson's proposal aside (but see n.44).

${ }^{4}$ I follow the dialectic of the novel feature debate in speaking of perceptual experiences. While the experience/subexperiential distinction does seem to be at play in explaining some of the intuitions I discuss, all that will matter for my arguments - particularly with respect to perceptual contents - is that the intuitions regarding the Aristotelian senses are what they are.

${ }^{5}$ The problem is especially salient for intramodal intentionalists, who claim that perceptual phenomenology supervenes on content + modality (e.g., Crane 2003, 2007; Lycan 1996). See Bourget (2017) and O'Dea (2006) for reasons why novel features pose a problem for intramodal intentionalism.
} 
Novel features are features of a perceptual experience that are not properly associated with any

Aristotelian sense (pre-theoretically understood) - vision, audition, touch, taste, or smell. ${ }^{6}$ Rather, they arise due to the operation of multiple senses working together. ${ }^{7}$ The relevant features are simple features of both the phenomenal character and representational content of the perceptual experience. When I speak of features, I will mean such simple features. (Sometimes the literature focuses solely on phenomenal features, other times both, and sometimes it is ambiguous between the two. I will consider both.) Note, also, that the Aristotelian senses are exteroceptive - they inform us about the external environment. Novel features - if there are any - are features of experiences by which we encounter our environment. The novel feature debate does not address features of interoceptive experiences, which inform us of states of our own body (such as hunger or pain). Novel features fall into two classes: ${ }^{8}$

i. Mere novel feature instances: Features of a perceptual experience that, while they can in some circumstances be associated with experience in just one sensory modality, only appear as a feature of the given experience due to the coordinated operation of at least two distinct sensory modalities.

\footnotetext{
${ }^{6}$ An anonymous reviewer for this journal pointed out the intriguing possibility of features that are mistakenly attributed to a modality. Such features are either: (a) features that ought to be associated with one modality but are, due to interaction of the two senses, apparently associated with another (see Spence (2016) on oral referral as a potential instance of this) or (b) features that aren't properly associated with any modality but are mistakenly thought to be. Features of type (a) would not be novel. Features of (b) would be novel. Making sense of mistaken feature associations will, of course, depend on a rule for associating features with modalities - just as understanding novel features does.

${ }^{7}$ And the modalities involved should suffice for the resulting experience to include the novel feature. Being a bit of pine wood is not a novel feature of an experience in which one picks up a stick and smells it to ascertain what sort of wood it is because, though one needs the olfactory input along with visual and/or tactile input for the experience to include that property in its contents, one will also need cognitive inputs (e.g., a PINE concept) for this property to work into the contents of the experience.

${ }^{8}$ This distinction was introduced and further developed by O'Callaghan (2014a, 2015, 2017). The term 'novel feature' is his.
} 
ii. Novel feature types: Features of a perceptual experience that are never associated with a modality; they can only appear as a feature of a perceptual experience due to the coordinated operation of at least two distinct sensory modalities.

Relations, the relata of which are perceived by different modalities, are the paradigm candidates for mere novel feature instances - e.g., the temporal relation between the sight of a baseball player's foot touching third base and the sound of the ball striking the third baseman's mitt used by an umpire to determine whether the runner makes it safely to third. Other candidates include cross-modally perceived gestalts, such as a rhythm articulated by alternating flashes and beeps. ${ }^{9}$ A standard candidate for a novel feature type is flavor, as distinct from taste, where olfactory receptors interact with stimulation of the taste buds and input from the trigeminal nerve (by which we sense, e.g., the coolness of the mint or the heat of a chili pepper) to deliver an experience that is not (supposedly) reducible to the contributions of each of these, individually. ${ }^{10}$ Another candidate is intermodal feature binding awareness - where features associated with different senses are experienced as bound together in a single perceptual object and the unity of the multisensory perceptual object is a feature of the experience (the putative novel feature). ${ }^{11}$

It has been suggested that there is a distinct flavor modality (e.g., Stevenson 2009). The proposed modality encompasses taste, smell, trigeminal tactile stimulation, and (in some cases) vision. Without ruling on the propriety of treating such a system as a distinct sensory modality, we can note that such a modality is not one of the Aristotelian senses in terms of which the novel feature debate is conducted. Nor do we have any pre-theoretical notion of this flavor sense - it is a

\footnotetext{
${ }^{9}$ O'Callaghan (2015) offers an extensive discussion of cross-modal gestalts in the context of the novel features debate; for a skeptical take see Spence (2015a).

${ }^{10}$ See, e.g., Connolly (2014) and O'Callaghan (2014a, 2014b, 2015, 2017, 2019) for (somewhat guarded) defenses of flavor as a novel feature type. For more on the various inputs to flavor perception see Auvray and Spence (2008), Spence (2015b). Again, Spence and Bayne (2014) offer reason to doubt that we have multisensory experiences, including the experience of multisensory objects. See also Spence and Frings (2020) for a discussion of one particular approach to multisensory feature integration.

${ }^{11}$ See especially O'Callaghan (2014a).
} 
theoretical posit. Any dispute between someone who claims that flavor includes novel features and one who claims that, while there are features involved in flavor experience beyond those supplied by taste, smell, and trigeminal stimulation, these are properly associated with a further (theoretical) flavor sense is merely verbal. This demonstrates the need for an agreed upon set of senses against which to evaluate novel features. In the novel feature debate, the Aristotelian senses play this role.

It is also important to mark a distinction made within this literature between cross-modal interactions between the (Aristotelian) senses and properly multisensory effects. Here is O'Callaghan on the matter: "one sense could causally but not constitutively impact another. And sensory processes might interact while every aspect of perceptual experience remains associated with some specific modality or another. So, despite all the cross-talk, conscious perceptual experience might remain modality specific" (2015, p. 134). Standard examples of such cross-modal interactions without novel features are recalibration effects of the sort that generate illusions like the McGurk effect and the ventriloquism effect.

In the McGurk effect, participants are presented with a video display of someone mouthing the syllable /ga/ onto which is dubbed the sound of someone pronouncing /ba/. Subjects report hearing /da/ even after they are made aware of the illusion. In the ventriloquism effect, a visual stimulus leads to a change in the apparent location of a sound. ${ }^{12}$ The effect gets its name from the experience of hearing a ventriloquist's voice as coming from her dummy's mouth (because the ventriloquist's mouth doesn't move but the dummy's does). To give a more modern example: when watching a movie while wearing headphones, we experience the voices of the characters on the screen as coming from the mouths of those characters, not as coming from somewhere between our ears (Callan et al. 2015).

\footnotetext{
12 The term 'ventriloquism effect' is sometimes used in a broader sense to include the spatial capture of audition by any other sense (see Caclin, Soto-Faraco, Kingstone, and Spence 2002). I will focus on the audiovisual ventriloquism effect described above.
} 
These illusions are the result of sub-experiential interactions between the senses. Such interactions resolve discrepancies between the senses when they carry conflicting information about some feature of the environment or allow one modality to inherit information from another modality, where the second modality has a greater acuity or is more reliable with respect to that class of information. For instance, vision has greater spatial acuity than audition - at least for stimuli projected onto the fovea (a cone-rich structure of the retina onto which the center of the visual field is focused). When auditory and foveal visual stimulation are attributed to a common source, the localization of the visual stimulus dominates audition, conferring the more precise visual location on the sound. This recalibration of information leads to a corresponding alteration in the phenomenology and content of the perceptual experience. In ordinary circumstances such recalibration tends to increase the reliability of the senses. However, in irregular circumstances such as those in which the illusions arise - these interactions can lead to errors.

The thought, widely endorsed in the novel features literature, is that such causal interactions do not result in novel features. ${ }^{13}$ A feature of a perceptual experience can still be associated with a single modality even when it is produced by more than one mechanism. If we classified features as multisensory strictly by the mechanisms that produce them, then nearly all the features of perceptual experience would be multisensory because causal interactions among the sensory mechanisms (insofar as we can assign them to pre-theoretical notions of the senses) are pervasive, as noted by O'Callaghan, above. ${ }^{14}$ While this is a perfectly legitimate practice - one common in the sciences - it

\footnotetext{
${ }^{13}$ Cf. Bayne (2014), with respect to contents. See Connolly (2014) for a reply. One might also push back on this claim by noting that cross-modal illusions do induce some meta-cognitive uncertainty about the reliability of the experience (Deroy, Spence, and Noppeney 2016). However, the uncertainty is not a feature of the first order experience itself; it is an assessment of the reliability of that first order experience. (We don't hear that there is uncertainty, we have uncertainty about what we hear.) Since novel features, if there are any, are features of the perceptual experience, metacognitive uncertainty won't undermine the intuition that cross-modal illusions don't produce novel features.

${ }^{14}$ See Driver and Noesselt (2008), Ghazanfar and Schroeder (2006), Schroeder and Foxe (2005), Shimojo and Shams (2001).
} 
deviates dramatically from that of partisans in the novel feature debate, with their reliance on the pretheoretical notions of the Aristotelian senses as the basis for classifying sensory features.

But, of course, to classify a feature as novel we will need some account of what it is for a feature (e.g., some particular color phenomenology) to be associated with a sensory modality (e.g., vision). As we saw with Bayne, above, philosophers debating the existence of novel features have generally relied on intuitive answers to this question (e.g., intuitions about the proper sensibles of a modality - those features that are perceivable by that modality and no others). But this approach will do no better, for the purposes of the novel feature debate, than the scientific approach detailed above. The appeal to such intuitions cannot settle the debate, since the features about which there is a dispute are precisely those about which intuitions diverge. If we are going to make any headway, we will need some other way of associating features with sensory modalities.

The challenge is to draw the distinction between a causal impact and a constitutive impact in such a way that these intuitions are respected. It is very appealing to think that it should be drawn in terms of the features that the modified modality might produce on its own. A causal impact won't the thought goes - exceed these bounds while a constitutive impact (yielding a novel feature) will. This is the thought behind O'Callaghan's proposed association rule, which we will examine shortly. Before we can assess that rule, we are going to need some criteria by which to judge it.

\section{Constraining the association rule}

Any acceptable association rule must meet certain constraints. For instance, if the rule is going to be useful in assessing whether or not there are novel features, (1) it will have to tell us, for any given feature and sensory modality, whether or not that feature is associated with that modality. And (2) the associations the rule delivers must conform to certain intuitive associations of features with modalities. A rule that associates colors with smell and not with vision is not a good rule. Also (3) the rule must not prematurely trivialize the novel feature debate: We must not be able to read the 
existence or non-existence of novel features right off the rule. (A rule with, e.g., a final clause, "otherwise the feature is to be associated with vision", does not satisfy this constraint.) And the novel features the rule allows should have independent theoretical interest. More generally, it must not render the debate unsubstantive.

The intuitive associations of the second constraint that I shall focus on concern (a) proper sensibles and (b) merely causal influences of one sense on another. With respect to (a), these are features that are intuitively associated with only one sensory modality. Color, for instance, is a feature of visual experience but not of olfaction, audition, touch, or taste. Similarly, pitch, timbre, and loudness are proper sensibles of audition; odors are proper sensibles of smell, and so on. The way I am using proper sensibles here applies both to features of content and phenomenal character. The relevant intuitions regarding (b)-type cases are just those concerning causal interactions, including cross-modal illusions, discussed in the prior section. A perceptual experience will contain no novel features due to these effects, not even mere novel feature instances. The impacted features are associated with the modality of the perceptual object that instantiates them - e.g., the illusory sound localization in the ventriloquism effect is associated with audition, not vision. These intuitions need to be respected if we are going to capture the pre-theoretical notions of the senses at play in the novel feature debate.

There is one more constraint, which will require a brief argument: (4) The association must be grounded in an appeal to the stimulation states of the sensory mechanisms of our modalities. In particular, the rule must appeal to the stimulation states of the mechanism of the modality with which the given feature is associated. ${ }^{15}$ Sensory mechanisms (the sensory organs, the portion of the nervous system connecting them to the brain, and the brain regions involved in processing inputs received

\footnotetext{
${ }^{15}$ If you are already satisfied that explanations of the contents and phenomenal character of perception ultimately need to be grounded in the physical (e.g., stimulation states of sensory receptors and subsequent neural processing), then you can skip the remainder of this section.
} 
from the sensory organs) and stimuli are two of the four axes along which theorizing about sensory modalities occurs. The other two are representational content/worldly features perceived and the phenomenal character of the perceptual experience - the very sorts of things novel features are. (These criteria were codified by Grice (1962).) So, for any sensory modality there will be a mechanism that responds to some characteristic stimulus (e.g., light), a set of phenomenal features, and a set of contents ascribed to that senses. Any attempt to associate features with sensory modalities straightaway in terms of the content of the perceptual experience or its phenomenal character (or the two in combination) will be inadequate for present purposes. Here's why:

We need to distinguish between mere groupings of (simple) features of a perceptual experience and groupings that amount to the set of features properly associated with a given sensory modality. This will require an account of what a modality is. For the purposes of the novel feature debate, the relevant modalities need to correspond to the pre-theoretical notions of the Aristotelian senses - vision, audition, taste, smell, touch. If we restrict ourselves to features of the experience in giving our account of the modalities, we will need to appeal to relations between these features. It won't do to merely stipulate associations - we would need to presume the very thing at issue in order to meet the first constraint. The difficulty is finding relations that don't appeal to anything beyond the features themselves and that don't beg the question either for or against novel features.

While some have argued that the proper approach to individuating the senses is to focus on content, phenomenology, or both, these proposal are not made with the novel features debate in mind. ${ }^{16}$ For their part, partisans in the novel feature debate have generally sidestepped the issue of individuating the senses (see the quote from Bayne, above). So, what might be said for the content and/or phenomenology approach to individuating the senses for the purposes of adjudicating the novel feature debate? ${ }^{16}$ The closest account we get is that of Richardson (2014). Richardson addresses causal influences of one sense on
another, but not the possibility of novel features. 
We might try individuating the Aristotelian senses in terms of discrete phenomenal continua - e.g., color or pitch - in terms of which a notion of phenomenal similarity could be spelled out. But doing so leaves us with too many senses - e.g., a pitch sense, a timbre sense, a color sense, etc. Or we might try an appeal to dependencies between such features, letting the senses be the collections of interdependent features. For instance, we only see colors if we see them qualifying some shape (or spatial expanse), so color and (a certain kind of) shape content/phenomenology belong to the same sense (vision). However, this won't tell you which non-dependent features ought to be associated with a sensory modality, but we need an association of such non-dependent features to ground our associations of the dependent features. ${ }^{17}$

Or we might try individuating the Aristotelian senses by phenomenology/content pairs. But we will still need a reason to pick certain pairings and not others. It is not the case that an experience with a particular content necessarily has a given phenomenology. For instance, we can have a perceptual experience with the content that there is a circle accompanied by either visual or tactile phenomenology. The question is why the one pairing should count as visual and the other as tactile. ${ }^{18}$ It won't be because the visual phenomenology is grouped along one phenomenal continuum and the tactile another, since we will have to include multiple continua in each sense (see above). We need some further reason to include/exclude the relevant continuum in/from the sense in question. We can't appeal to dependences to group the relevant continua into a sense (e.g., one can only see the color of the circle if one has the visual sort of circle phenomenology) without some prior rule for

\footnotetext{
${ }^{17}$ And there are features that, while not seeming to ground any further associations, are non-dependent and are intuitively associated with an Aristotelian sense - e.g., silence is associated with audition and empty space is associated with multiple senses: I can see the space between two objects, or I can feel the empty space in front of me when I have my hand about.

${ }^{18}$ The point applies to experience with non-perceptual contents, too. I could be thinking about circles (or whatever else) while looking at a blank wall. Why shouldn't the intuitively non-perceptual content get paired with some perceptual phenomenology and, on the strength of that pairing, be associated with one of our sensory modalities? Presumably we distinguish perceptual contents from non-perceptual contents because the former, and not the latter, are the result of a perceptual mechanism. But this has us appealing to mechanisms, so it can't be the answer for the content/phenomenology pairing approach.
} 
grouping the non-dependent features. But there don’t appear to be any other relations between features of a perceptual experience that could make the difference of a sense. ${ }^{19}$

So we can't give an adequate account of the sensory modalities (for present purposes) in terms of just the content and phenomenal character of an experience. Fortunately, we can appeal to Grice's other two criteria, mechanisms and stimulation, in formulating our association rule individuating the senses by sensory mechanisms and using the stimulation states of the mechanisms as the basis of associations of features with modalities - namely, the modalities responsible for the production of those features. ${ }^{20}$ Hence the fourth constraint on our association rule.

\section{O'Callaghan's association rule and some morals drawn from it}

\subsection{O'Callaghan's rule}

O'Callaghan has offered an association rule that, at first blush, seems to satisfy this fourth constraint: "for each perceptual experience of a given modality, the [features] it instantiates that [are] associated with that modality on that occasion [include] only that which a corresponding [unimodal] experience of that modality could have" (2014b, p. 152). ${ }^{21}$ He defines "corresponding experiences" as experiences resulting from an equivalent stimulus (2014b, p. 152). O'Callaghan understands unimodal experience in terms of mechanisms, suggesting that we get a fix on a unimodal experience by considering what is left once we subtract the contributions of the mechanisms associated with the other sensory modalities (2014b, p. 145). More formally, for an instance of a feature F of a perceptual experience E resulting from a stimulus R:

\footnotetext{
${ }^{19}$ If you are inclined to respond with an appeal to the relative determinacy of visual v. tactile shape discrimination, substitute this example: We can feel and see the location of a pin prick on our fingertip.

${ }^{20}$ Since mechanisms are partially defined in terms of their response to stimuli, there is no disentangling the two. There are proposals that don't rely, directly, on Grice's criteria (esp. Nudds 2004) but appeal to conventions - e.g., those features count as visual that we conventionally associate with vision. However, such accounts - by their own admission - won't deliver unambiguous verdicts in the controversial cases.

${ }^{21}$ O'Callaghan's formulation focuses only on phenomenal features. I adjust it here to include content.
} 
(OCR) That instance of $F$ is associated with a given modality if, and only if, a corresponding instance of $\mathrm{F}$ could be a feature of a unimodal experience $\mathrm{E}$, in that modality, resulting from stimulation by $\mathrm{R}^{22}$

So, the smell of coffee brewing, when part of a perceptual experience including the sight of bread in the toaster, the feel of a jar of jam in your hand, the sound of coffee percolating, etc., will belong to olfaction because, were we to isolate olfaction, ruling out any input from the other modalities, and present it with exactly the same scene, the resulting experience would include the smell of the coffee brewing. But the sound of the coffee percolating would not be associated with olfaction because olfaction would presumably not, in the presence of that same total stimulus, deliver the sound of percolating coffee. It is clear what OCR excludes: If a feature instance is to be assigned to a modality, its production cannot require concurrent stimulations outside the characteristic sensory inputs to that modality. But what about (i) past stimulations in another modality, as when one now "sees" the heat of an ember thanks to learned correlations between heat and a red glow? And what about (ii) inputs that are not stimulations at all, such as an innate Kantian spatial form of intuition? Until these questions are answered OCR cannot satisfy our first constraint. ${ }^{23}$ It does not tell us for any given feature instance and for each modality whether or not the feature instance is associated with that modality. ${ }^{24}$ With respect to (i), for instance, we learn to hear the elevation of a

\footnotetext{
${ }^{22}$ All the proposed rules should be read as relativized to normally functioning sensory mechanisms. Notice, also, that the rules are formulated in terms of feature instances. A novel feature type will be one with no instances that are associated with any modality. We can say that a feature type is associated with a modality if some unimodal experience of that modality instantiates that feature. Finally, recognize that the association relation cannot be a function - we must leave open the possibility that a feature is associated with more than one modality if we are to accommodate common sensibles. It might be that the association relation is a function for phenomenal features, but that is a substantive philosophical thesis that is more often assumed than argued for.

${ }^{23}$ To be fair, O'Callaghan (2014b) does discuss the problem of learned correlations, drawing a distinction between two kinds of unimodal experience: pure and mere experiences of a modality. The former are perceptual experiences had by a creature whose past and present experiences have only been in that modality. The latter are those had by a creature whose experience is presently, but not historically, restricted to that modality. O'Callaghan inclines towards 'yes'. Others - most notably Strawson (1959) in his discussion of sounds and a 'no space world' - have said 'no'. De Vignemont's (2014) multimodal account of bodily awareness also depends on a 'no' answer. O'Callaghan does not discuss stimulus-independent inputs.

${ }^{24}$ One might also worry that there is no uncontroversial way of individuating the senses and, so, no way of identifying what, say, vision is - let alone what features should be associated with it (Coady 1974; Fulkerson 2014c; Gray 2013; Grice 1962; Heil 1983; Keeley 2002; Macpherson 2011b, 2011c, 2014; Matthen 2015; Nelkin 1990;
} 
sound by correlating the effects of the outer ear on the waveform of the sound - which differ with elevation - with visual input regarding the elevation of sound sources. Whether or not we include this correlation as part of the input to the unimodal experience will determine whether or not we should associate sound elevation with audition according to OCR.

The problem is even worse for (ii). Whatever could have been supplied (in terms of content or phenomenal character) by the other senses could be supplied by such inputs. There could be, e.g., an innate correlation between waveform effects and sound elevation that does just what the learned correlation discussed above does. And if that is possible, subtracting vision (or any other modality) won't shed any light on the deliverances of unimodal audition according to OCR. Likewise for all the other modalities. For instance, there could be an innate correlation between the smell of coffee brewing and the sound of a percolator, in which case OCR would associate the sound with olfaction. (I will have more to say about problems resulting from the use of 'could' below.)

The lesson is that we will have to take a stance on what is and is not actually part of/an input to the mechanism responding to the stimulus and giving rise to the experience (including any learned correlations and non-sensory inputs) before we can successfully apply an association rule based in an individuation of the senses by those mechanisms. Before proceeding to further attempts at an association rule, some remarks about these sensory mechanisms are in order.

\subsection{Comments regarding mechanisms and modalities}

Nudds 2004; Roxbee-Cox 1970). However, the cases considered in the individuation of the senses literature concern determinable senses - the sort of senses that might be shared by creatures with differing sensory mechanisms and capacities associated with that sense (e.g., a sense of vision that can be had by humans, bees, and pit vipers). The novel feature debate, on the other hand, concerns a characterization of our experiences. If we focus just on the (relatively) determinate senses that we, as a species, share (as O'Callaghan does in his discussion), we might get a workable individuation of the Aristotelian senses. (I borrow the determinate/determinable sense distinction from O’Callaghan (2019, chapter 6).) 
The preceding discussion should alert us to the fact that we do not have uncontroversial intuitions about the exact extent of the sensory mechanisms of the Aristotelian senses. ${ }^{25}$ This suggests that there are multiple intuitively acceptable ways of demarcating these mechanisms. This poses a problem for the novel feature debate because differences in demarcation will lead to differences in feature associations. ${ }^{26}$ And these differences are most likely to arise with respect to the controversial feature association, including those concerning putative novel features. For instance, one acceptable demarcation might associate contested flavor features with taste while another doesn't associate it with any of our Aristotelian senses. But the debate presumes that there will be an unqualified answer to the question 'are there novel features?' We will need a criterion for selecting a preferred demarcation of the mechanisms of a given Aristotelian sense if we are to get our unqualified answer. Here we can press our association rule into service. The idea is that candidate demarcations will be evaluated with respect to their ability to deliver the right verdict regarding our guiding feature association intuitions from constraint (2), among others. (These other intuitions - call them secondary intuitions - will be intuitive feature associations that we would like to retain if we can, but which we are willing to give up to save more central intuitions.) In order to do this, though, we need to settle on the right association rule - and that requires having clearly demarcated mechanisms for the rule to operate on. We need the rule to pick the demarcation, but we need the demarcation to pick the rule.

The key, to resolving this difficulty, is finding some equilibrium between intuitions regarding feature associations and intuitions regarding the mechanisms of the Aristotelian senses. Returning to

\footnotetext{
${ }^{25}$ Indeed, it isn't entirely clear whether or not cognitive inputs ought to be included in the sensory mechanisms, not least because it isn't clear where the line between perception and cognition should be drawn. If cognitive resources are required in order to give rise to experience, then they will certainly be involved in the relevant mechanisms. Specifying which cognitive resources are included will have ramifications for the debates over rich/thin contents of perceptual experience and cognitive penetration.

${ }^{26}$ In developing his sensory pluralism, Fulkerson (2014c) notes that different individuations (chosen for different purposes) will give different results for different experiences. He doesn't question that there will be some individuation of the senses that will be acceptable for answering the general question that is in dispute in the novel feature debate. (See Macpherson 2011b, 2011c for a similar sensory pluralist view.)
} 
our second constraint on the association rule - that it must satisfy certain guiding feature association intuitions - we can add the qualification: provided the notion of a modality that you feed into it satisfies guiding intuitions about that modality. It is no mark against an association rule that it associates colors with olfaction if the relevant notion of olfaction includes retinal stimulation.

Fortunately, though they don't decide what to say about inputs of type (i) and (ii), our intuitions concerning the mechanisms of the Aristotelian senses do not leave us entirely at sea. For instance, it is uncontroversial that each of those senses has primary receptors (e.g., retinas for vision, the basilar membrane for audition, etc. ${ }^{27}$ ) that are tuned to a particular class of energy (e.g., light, pressure waves, etc.) and that the primary receptors of a given sense are not part of the mechanisms of the other senses. ${ }^{28}$ These intuitions are linked to, but not dependent on, the feature association intuitions of constraint (2). Regarding the intuition that the proper sensibles are associated with only one sensory modality, color is a proper sensible of vision because color is the sort of thing that is only detectable by the primary receptors of vision. Similarly, the paradigmatic instances of recalibration effects, including the McGurk and ventriloquism effects, require stimulation of the primary receptors of multiple modalities.

(It has been shown that mental imagery can induce cross-modal illusions - e.g., that an imagined visual stimulus can induce the ventriloquism effect (Berger and Ehrsson 2013). However, this does not impact the primary receptor intuition any more than our ability to imagine a color while receiving no visual stimulation does. The primary receptor intuition is linked to the proper sensible and recalibration effect intuitions only with respect to their paradigmatic instances - i.e., those

\footnotetext{
${ }^{27}$ Things get a little trickier for the other senses but, I think, not unbearably so.

${ }^{28} \mathrm{We}$ might think that this gives us an alternative rule: A feature of a perceptual experience is associated with a given modality just in case we lose those features of the experience in a corresponding situation differing only in that there is no stimulation of the primary receptor. However, this test won't work. Consider the McGurk effect: If we omit stimulation of the basilar membrane (primary auditory receptor), we lose /da/ phenomenology (i.e., the sound of someone pronouncing /da/), so this feature is associated with audition. But if we omit retinal stimulation, we also lose that phenomenology, so it will also be associated with vision. But intuitively, /da/ phenomenology should only be associated with audition.
} 
resulting from occurrent stimulation of the senses involved. And it does not depend on these feature association intuitions even in the paradigmatic instances. If anything, the order of explanation goes the other way around. The primary receptor intuition is not affected at all by the existence of nonparadigmatic instances of the effects, such as those involving mental imagery. $)^{29}$

So, any acceptable candidate for the preferred demarcation of the sensory mechanisms to be used in adjudicating the novel feature debate will need to conform to our intuitions regarding primary receptors of the Aristotelian senses. With one further (safe) assumption - namely, that we don't have stimulus-independent resources for duplicating the information about our environment that the primary receptors would capture in a given circumstance, were they active (whether or not they are) - we can assess how our rules fare with respect to our intuitions concerning proper sensibles and the McGurk and ventriloquism effects using a general notion of an acceptable demarcation - a placeholder demarcation on which the primary receptor condition and the safe assumption are satisfied.

The goal is to find a rule that can deliver these intuitions for the general notion of an acceptable demarcation of the mechanisms of the Aristotelian senses. Once we have the rule in hand, we can use it to find our preferred demarcation by testing the acceptable candidate demarcations with respect to their ability to deliver our secondary intuitions. In short, we find our rule using the generalized acceptable demarcations of the Aristotelian senses, and then we us our rule to find the preferred demarcation of the mechanism of each of those senses.

One might worry that, even with an association rule in hand, there will still be equally good candidates for the preferred demarcation that deliver different verdicts with respect to specific novel features. Be that as it may, without an association rule there is no hope for a resolution to the novel

\footnotetext{
${ }^{29}$ Such imagery is another non-stimulus input of the sort discussed in relation to OCR, above. It remains an open question whether or not the mechanisms of imagery ought to be included in those of one of our Aristotelian senses and, relatedly, whether or not imagery is properly thought of as perceptual. Settling the question will have consequences for applications of the association rules to follow.
} 
feature debate. With a rule some hope remains, so it behooves us to make the effort to find an adequate association rule.

We have already seen that the rule must operate on clearly demarcated sensory mechanisms, so the dimensions along which the proposed rules can vary are: what counts as a result of the stimulation states of a mechanism and how much we allow the stimulus to deviate from the actual stimulus in a counterfactual unimodal experience. In what follows I consider a series of modifications to OCR, each of which differs from its predecessor by a minimal adjustment in one or the other of these dimensions, thereby covering all the plausible ways of formulating an association rule. As we shall see, none of these attempts succeed, so the worry above never gets off the ground. (Tables summarizing the rules and how they fare with respect to the four constraints are included in the appendix.)

\section{Revising the association rule}

\subsection{Could and would in determinate sensory mechanisms}

Let's first modify OCR so that it will distinguish between our candidate demarcations of the mechanisms of a given sense. For an instance of a feature F of a perceptual experience E resulting from the stimulation of the sensory mechanisms $\mathrm{M}_{1}, \ldots, \mathrm{M}_{\mathrm{n}}$ by a (total) stimulus $\mathrm{R}$ :

(RR1) That instance of $F$ is associated with the modality individuated by $M_{m}$ if, and only if, were just $\mathrm{M}_{\mathrm{m}}$ stimulated by $\mathrm{R}$, a corresponding instance of $\mathrm{F}$ could be a feature of the resulting experience E'.

According to RR1 we will be able say that, if $\mathrm{M}_{\mathrm{m}}$ is an auditory mechanism that includes learned correlations by which waveform effects can convey information about elevation, the experience of the elevation of a sound will be associated with audition. If $\mathrm{M}_{\mathrm{m}}$ does not include the learned correlation (or an innate proxy thereof), sound elevation will not be associated with audition. That is an improvement over OCR. 
However, the phenomenal features that could result from a unimodal experience under equivalent stimulation will not be as constrained as we want. Some deaf musicians, for instance, are able to distinguish pitch by tactile sensation of a vibrating surface (the surface being vibrated by or being the source of a sound wave). The resulting experience - or any tactile experience of a vibrating surface, for that matter - could have the phenomenal character we associate with pitch rather than the characteristic feel of a vibrating surface. But then RR1 associates features that ought to be proper sensibles (e.g., pitch phenomenology) with more than one modality (e.g., audition and touch), in violation of one of our guiding intuitions from constraint (2) on an acceptable association rule. ${ }^{30} \mathrm{RR} 1$ is too promiscuous in its associations.

We can avoid this promiscuity by considering only what would result from the actual, determinate sensory mechanisms of the subject:

(RR2) That instance of $F$ is associated with the modality individuated by $M_{m}$ if, and only if, were just $\mathrm{M}_{\mathrm{m}}$ stimulated by $\mathrm{R}$, a corresponding instance of $\mathrm{F}$ would be a feature of the resulting experience E'.

This corrects for our tactile pitch phenomenology. We wouldn't have pitch phenomenology arising from tactile vibrations given the sensory mechanisms we actually have (on an acceptable demarcation of the tactile mechanism). However, given the restriction to the exact stimulus, $\mathrm{R}$, in the counterfactual situation, there will be no way of capturing intuitions regarding recalibration effects. If the mechanism delivers a given feature only because of the influence of another mechanism, then that feature would not be contributed by the unimodal mechanism under that stimulation.

For example, we cannot attribute any phoneme content/phenomenology to audition in the McGurk effect because the unimodal auditory mechanism would not, under equivalent stimulation

\footnotetext{
${ }^{30}$ While there is some evidence, from a select number of long-time users of sensory substitution devices, that stimulation in one modality can result in some rudimentary phenomenology characteristic of another, this is due to recruiting resources of the latter modality to the processing of input to the former, in which case we are dealing with a different mechanism (one including the recruited resources) than the one with which we began (the ordinary touch mechanisms). The point here is that no acceptable demarcation of the ordinary mechanism of touch has, as a matter offact, the ability to produce pitch phenomenology on its own, though it is conceivable that it could.
} 
result in $/ \mathrm{da} /$ content/phenomenology. That requires stimulation of the primary receptors of the visual mechanism as well. We will need to loosen the association rule if we are to capture the guiding intuition that the altered phoneme phenomenology/content in the McGurk effect is auditory.

\subsection{Loosening constraints on the stimulus}

Perhaps we should loosen the restriction on the stimulus to something like 'a substantially similar stimulus' (assuming this can be made sufficiently precise to avoid violating our first constraint). This would give us, for an instance of a feature $\mathrm{F}$ of a perceptual experience E resulting from the stimulation of the sensory mechanisms $\mathrm{M}_{1}, \ldots, \mathrm{M}_{\mathrm{n}}$ by a (total) stimulus $\mathrm{R}$ :

(RR3) That instance of $F$ is associated with the modality individuated by $M_{m}$ if, and only if, there is some stimulus $R^{\prime}$ that is substantially similar to $R$ and, were just $M_{m}$ stimulated by $R$ ', the resulting experience $\mathrm{E}^{\prime}$ would have a corresponding instance of $\mathrm{F}$.

The idea here is to associate features with the mechanisms that would produce them under some similar stimulation, privileging unimodal mechanisms. ${ }^{31}$ For instance, any acceptable auditory mechanism is capable of giving rise to an experience representing / da/ and providing the phenomenal features we associate with the pronunciation of that syllable. So, we can class /da/ experiences as auditory, even when they are produced by a multimodal mechanism, provided the stimulus that would result in unimodal /da/ experience counts as substantially similar to the stimulus of that multimodal mechanism.

But there is a problem. Consider the following: I see what looks like a speckled (flat) wall while also feeling that the wall is rough with my hand. Had the bumps on the wall been slightly

\footnotetext{
${ }^{31}$ Alternatively, you might think that a feature will be associated with a modality just in case it is one of the disjuncts in the disjunction of possible features resulting from the actual stimulus state of that modality's mechanism plus whatever effects can be generated on its output by the other mechanisms under a range of stimulation. Such a rule will need a non-question begging means of identifying the experiential results of a causal influence of one mechanism on another to avoid associating every feature of the experience with each modality. This will require a fuller specification of the dependencies of phenomenal features on informational content carried by the sensory mechanisms. See $\S 5.3$ for reasons to think this won’t help adjudicate the novel feature debate.
} 
higher or had the lighting come from a slightly different angle, the wall would have visually appeared rough. RR3 will correctly associate the content that the wall before me is rough with touch; but it will also associate that content (incorrectly) with vision because, were I presented (in a unimodal visual experience) with a substantially similar stimulus that differed just in the height of the bumps or the angle of the light, I would visually experience the content that the wall is rough ${ }^{32}$ On the other hand, RR3 will not ascribe tactile roughness phenomenology to vision because the tactile phenomenology isn't the sort of phenomenology that (any acceptable notion of) vision would have from our substantially similar visual stimulus. The point is that RR3 does not do well with respect to the contents of the experience, at least where the contents concern common sensibles. ${ }^{33}$ Fortunately, there are other approaches to identifying the content of a perceptual experience, ${ }^{34}$ so I will set contents aside and focus on an association rule that works for phenomenal features for the remainder of the paper.

However, RR3 also gives us the wrong results with respect to the phenomenology of ventriloquism effect experiences because the effect does not merely relocate the sound, it makes the localization more precise. And this is not restricted to the ventriloquism effect. In general, sound localization phenomenology resulting from the stimulation of just the auditory mechanism will not be as refined as sound localization phenomenology resulting from the auditory mechanism when aided

\footnotetext{
32 This result doesn't depend on any resolution of the conflict between information carried by the visual and tactile mechanisms, as in work on the visual dominance of touch (Rock and Victor 1964; Rock and Harris 1967). It depends on the fact that there is a possible stimulus similar to the actual stimulus that would make a content accessible to vision that is only accessible through touch in the actual experience - i.e., the visual mechanism plays no part in contributing that content to the original experience.

${ }^{33}$ This is not to say that the test could not be salvaged for content. Perhaps one could make a response along the lines of Nudds's (2009) distinction in the determinateness of experiences of common sensibles perceived with different modalities or an appeal to the total content characterizing the experience in which the content in question is embedded. Or perhaps we could try to restrict 'substantially similar' to rule out these cases. But these will require taking on theoretical commitments that might not pan out.

${ }^{34}$ E.g., naturalized accounts of intentionality such as Dretske (1981, 1988, 1995), Millikan (1984), Neander (2017), Papineau (1984), Shea (2018).
} 
by foveal visual input. ${ }^{35}$ But then, if we just consider an experience resulting from substantially similar stimulation of the auditory mechanism alone, we won't get sound localization phenomenology as in the actual experience. We will get a much less precise localization. So, the localization of the sound will not be associated with audition according to RR3, contrary to our intuitions. The same is true of every recalibratory cross-modal mechanism in which one of the mechanisms is more finely tuned than the other but we want to associate the resulting (phenomenal) feature with the latter modality. ${ }^{36}$

It will be no help to loosen our restriction on the stimulus further, by dropping the similarity requirement and leaving the stimulus unconstrained:

(RR4) That instance of $F$ is associated with the modality individuated by $M_{m}$ if, and only if, there is some stimulus $\mathrm{R}^{\prime}$ such that, were just $\mathrm{M}_{\mathrm{m}}$ stimulated by $\mathrm{R}$ ', the resulting experience $\mathrm{E}$ ' would have a corresponding instance of $\mathrm{F}$.

We still get the same result with respect to vision-assisted sound localization because no stimulus impinging on the auditory mechanism alone can give the fine-grained sound spatialization that arises from the recalibration of sound localization by foveal visual input to the auditory mechanism.

Furthermore, this rule associates all the common sensibles in a given experience with every modality that is able to give rise to them, whether or not they do so in that experience. RR4 is decidedly worse than RR3.

One might be inclined, at this point, to bite the bullet and abandon our guiding intuitions regarding the ventriloquism effect and other precision increasing sensory interactions. However, it is unclear why we should allow the retreat to substantially similar stimulation to save the McGurk intuition but not allow a retreat to a substantially similar, but less precise, result to save the

\footnotetext{
${ }^{35}$ See Battaglia, et al. (2003), King (2009), and especially Bizley and King (2008). See Blauert (1997, pp. 193-196) for an overview. For a presentation of the most precise spatial information available to audition from purely auditory cues, see Shinn-Cunningham, et al (2000).

${ }^{36}$ Such recalibration for greater precision does not presuppose greater accuracy, as the case of the ventriloquism effect should make clear.
} 
ventriloquism intuition. Pending a compelling reason for the asymmetry, we ought to see if we can revise our association rule so that it captures both intuitions. The next section attempts to spell out just how we ought to do this.

\subsection{Loosening what counts as a result}

Perhaps there is some profit to be gained by considering the differences between the ventriloquism effect and a case where RR3 arguably gets things right with respect to novel features, flavor. Flavor experience (allegedly) contains novel feature types whereas the ventriloquism effect experience merely evinces increased precision with respect to a unimodally available feature type (sound localization phenomenology). The flavor/taste distinction is, supposedly, a difference in kind. The ventriloquism effect (and ordinary visually enhanced auditory localization, generally) reveals a difference in degree.

But RR3 cannot capture this distinction because it is bound by those features that the unimodal mechanism - however demarcated - would deliver on its own. We have no room left to maneuver with respect to either stimulus or mechanism. This suggests that we need to loosen our requirement on the features that would result from substantially similar stimulation. But we will need to do so in a way that does not wind up attributing too much to the modality. ${ }^{37}$ A first pass at the required modification would be to associate features of a perceptual experience with a modality that either would produce them, under some substantially similar stimulation, or would produce a feature that is a determinate of the same determinable as the initial feature - a determinable being a relatively indeterminate property (e.g., color) and a determinate of a determinable being a more specific property in the range of the determinable (e.g., blue). Determinates of a given determinable can,

\footnotetext{
${ }^{37}$ Having already seen that the rule must operate on determinate mechanisms and that no loosening of the variation in the simulation between the actual and the counterfactual situations will, on its own, get us an acceptable rule, this is all that is left to us.
} 
themselves, be determinables with yet more specific determinates (e.g., azure). (The appeal to determinates/determinables is a more general way of capturing difference in degree/kind alluded to above.) So, for an instance of a feature $\mathrm{F}$ of an experience $\mathrm{E}$ resulting from the stimulation of the sensory mechanisms $\mathrm{M}_{1}, \ldots, \mathrm{M}_{\mathrm{n}}$ by a stimulus $\mathrm{R}$ :

(RR5) That instance of $F$ is associated with the modality individuated by $M_{m}$ if, and only if, there is some stimulus R' that is substantially similar to R and, were just $\mathrm{M}_{\mathrm{m}}$ stimulated by R', the resulting experience E' would either:

(a) have a corresponding instance of $\mathrm{F}$ or

(b) have a corresponding instance of a feature F' that is a determinate of the same determinable as F.

RR5 does save the ventriloquism intuition, but it runs afoul of one of our other constraints: (b) introduces an opportunity for features to become associated with a modality despite the mechanism of that modality not being involved in the production of that feature. This is a straightforward violation of the fourth constraint.

To illustrate the problem, it will be useful to appeal to a hypothetical case that allows us to abstract away from the difficulties posed by our own, complicated perceptual systems. Imagine a race of aliens very much like us except that they have evolved such that they ordinarily experience a form of lexical-gustatory synesthesia. When they hear names in their home language, they have concurrent taste phenomenology of some sort of sugary soft drink, a different one for each name. (They evolved to have this synesthesia because it is, for them, conducive to social bonding. ${ }^{38}$ ) For the purposes of this example, we will include whatever mechanisms are necessary to produce the synesthetic taste phenomenology in this alien race's auditory mechanism. (This will still count as an acceptable demarcation of audition because stimulation of the primary receptors of their gustatory mechanism is not necessary for the synesthetic taste phenomenology.) Furthermore, let us stipulate that there are no other relevant interactions between these and the other senses complicating the case.

\footnotetext{
${ }^{38}$ Also note that, for these aliens, taste - at least in the range of tastes of sugary soft drinks - is not a proper sensible. Nothing in the example depends on this, nor does this case rely on the feature association intuitions of constraint (2).
} 
In sum: these aliens have auditory mechanisms that produce taste phenomenology in a narrow range (sugary soft drink tastes) when they hear names, and there are no other relevant interactions between their senses in producing their auditory and gustatory experiences. Otherwise they are just like us.

Now suppose that one of these aliens, Lexi, visits earth, settles in California, learns English and assimilates with human society there. Furthermore, 'Joan' turns out to be a name in Lexi's home language, one resulting in a synesthetic experience of the taste of root beer. No other English words or names are names in her language.

One day Lexi is drinking coffee when her friend Gus says, "June is always gloomy in Los Angeles". (In terms of RR5, this total scene, including the coffee drinking and Gus's utterance, is the stimulus R). When Lexi hears "June" she has no synesthetic taste phenomenology - it is not one of the names that triggers her synesthesia. But she does experience the taste of coffee (RR5's F, in this case) because she is, in fact, drinking coffee. However, the sound of "June" is very similar to "Joan". ${ }^{39}$ So there is a stimulus (R') - namely, Gus uttering "Joan is always gloomy in Los Angeles" while everything else remains as it is in $\mathrm{R}$ - that is substantially similar to the original stimulus and that would result, under unimodal stimulation of Lexi's auditory mechanism, in a corresponding feature $\left(F^{\prime}\right)$ - the taste of root beer - that is a determinate of the same determinable (phenomenal taste) as the coffee taste she is actually experiencing. Therefore, all the conditions on the right side of RR5 are satisfied, delivering the verdict that the coffee taste is associated with audition. Yet Lexi's auditory mechanism has nothing to do with her tasting coffee. Her synesthesia never produces the taste of coffee. So this verdict violates constraint (4). ${ }^{40}$

Of course, one could respond that we have latched on to the wrong determinable. The relevant determinable is just the range of tastes that Lexi's auditory system, when properly

\footnotetext{
${ }^{39}$ If you don't find 'Joan' and 'June' sufficiently similar, substitute the French (and alien) name 'Jeune' for 'Joan'.

${ }^{40}$ This conclusion follows even if there are no creatures that work according to the stipulations we have introduced to describe Lexi. The point is simply that the rule is not adequately tied to the mechanisms that produce the features to be associated.
} 
stimulated, can produce in her - namely, sweet soft drink tastes. That would rule out coffee. But this just points to a further worry; namely, that we can carve determinables more or less finely, with different ways of doing so giving different associations according to RR5. We need a way of picking out the relevant determinable.

The response in the preceding paragraph - that the objection relies on using the wrong determinable - is based in the intuition that the relevant determinable is fixed by the range of the mechanism in question (e.g., the range of taste phenomenology that Lexi can experience from stimulation of her auditory mechanism). Adding this restriction to RR5 would certainly help resolve the violation of the fourth constraint, but hewing too closely to the capacities of the mechanism in question is what caused our problem with the ventriloquism effect (or any visually assisted auditory localization) with respect to RR3 and RR4: The auditory mechanism lacks the spatial resolution of (foveal) vision-supported auditory localization, so (foveal) vision-supported localization phenomenology will fall outside the range of the relevant determinable. That means that we cannot associate the localization of the sound with audition, contrary to our intuition. If we don't restrict ourselves to the range of the determinable deliverable by the mechanism in question, we risk throwing ourselves back on the problem of selecting the relevant determinable (as we just saw). There is a middle way: restricting the determinable to the range of features that the subject's sensory mechanisms can, individually, supply. ${ }^{41}$ However, this won't fix RR5's rulings in Lexi's case. The taste of coffee is deliverable by one of Lexi's sensory mechanisms; namely, the gustatory mechanism. We are going to need a more nuanced approach.

In particular, we need a notion of feature inheritance - conditions under which a modality can take on a feature of another modality. With the notion of inheritance properly spelled out, we will be able to fix on the relevant determinable (according to our middle way) and accommodate the

\footnotetext{
${ }^{41}$ To drop the restriction to the deliverances of the individual mechanisms would be make novel features impossible, in violation of the third condition on our association rule.
} 
ventriloquism effect and Lexi intuitions. The idea is that, in the ventriloquism case sound localization is inherited but in Lexi's case coffee taste is not. So inheritance constrains the appeal to shared determinables in clause (b), bringing it in line with constraint (4).

In a proper instance of inheritance, the original stimulus $(\mathrm{R})$ would not result in a unimodal experience of the inherited determinate $(\mathrm{F})$ in the inheriting modality. We can narrow this further, given that clause (a) will capture any instances of the determinate that would result from some substantially similar stimulation: Inheritance involves determinates of a unimodally accessible determinable that wouldn't result from a substantially similar stimulus (R'). Furthermore, inherited features are inherited from some other mechanism that can produce them in a unimodal experience. ${ }^{42}$ For example, when the visual localization of a trumpet is bound to an auditory object (the sound of the trumpet) in (foveal) vision-assisted sound localization, the localization - which is too precise to have been supplied by a unimodal auditory experience - is supplied by vision and inherited by audition. By contrast, Lexi's coffee taste phenomenology is not inherited by audition - it is just a straightforward result of her taste mechanism.

It is not necessary that the inherited feature be a feature of an object in the parent modality in the original experience (i.e., that (a) is satisfied for the parent modality), though this is typically so. Restricting inheritance in this way would present problems. First, it can't accommodate the intuition that hemifield neglect patients experiencing the ventriloquism effect on their neglected side inherit the localization of the sound from vision (because there is no corresponding visual experience). (Hemifield neglect patients are susceptible to both the McGurk and the ventriloquism effects even when the visual stimulus is presented to their contralesional side and so, presumably, without any accompanying visual phenomenology (Bertelson et al. 2000; Leo et al. 2008; Soroker et al. 1995a,

\footnotetext{
${ }^{42}$ For the sake of thoroughness, we should acknowledge the possibility of chains of inheritance - e.g., one modality inheriting a feature from a mechanism that inherited the feature from yet a third mechanism. At some point chains of inheritance will need to bottom out in a mechanism that has non-derivatively supplied the feature.
} 
1995b).) Also, some recalibration effects persist even after the contributor modality stops receiving stimulation. $^{43}$

The appeal to shared determinables also constrains inheritance. In particular, it captures the idea that we don't inherit features arbitrarily - e.g., we don't have experiences of green sounds and circular smells. And the restriction to a corresponding instance of a shared determinable captures the idea that instances of inheritance involve the inherited feature $(F)$ overwriting a feature (F') of the given perceptual object that would have been produced under substantially similar unimodal stimulation. This overwriting follows from the fact that the feature inherited $(\mathrm{F})$ is a determinate of the same determinable as is the unimodally available feature (F'). Determinates of the same determinable are incompatible - an object cannot be both entirely red and entirely blue. Adding this notion of inheritance to RR5, we get:

(RR6) That instance of $F$ is associated with the modality individuated by $M_{m}$ if, and only if, there is some stimulus R' that is substantially similar to R and, were just $M_{m}$ stimulated by R', the resulting experience E' would either:

(a) have a corresponding instance of $\mathrm{F}$ or

(b) have a corresponding instance of a feature, F', that is a determinate of the same determinable as $\mathrm{F}$, provided that

(i) $\mathrm{M}_{\mathrm{m}}$ inherits the instance of $\mathrm{F}$ from some other mechanism $\mathrm{M}_{\mathrm{k}}$ in $\mathrm{E}$.

RR6 seems to get the verdict right in the ventriloquism effect - we will say exactly the same thing about it as we did about the localization of the sound of the trumpet, above. And it certainly gets the right verdict with respect to Lexi's case. The taste of coffee is not inherited by audition - Lexi doesn't experience the coffee taste because her auditory mechanism inherited the feature from her gustatory mechanisms. She tastes the coffee as a result of the operation of her gustatory mechanism, itself. Notice, though, that we can say this in Lexi's case because we stipulated that there were no complicating interactions between the senses. Real life cases will require empirical facts to determine

\footnotetext{
${ }^{43}$ How the coordination of perceptual objects across modalities actually goes will be addressed in more detail below.
} 
when a mechanism does and doesn't inherit a feature from another. And this is going to lead to a problem for RR6.

Consider the justification on offer for treating the ventriloquism effect localization phenomenology as auditory: A feature (localization) is bound to an auditory object (a sound), where that feature was inherited from vision. Without the empirical details regarding the interactions of the visual and auditory mechanisms, we have to fall back on our intuitions about which perceptual objects belong to which sensory modalities. But we (intuitively) identify perceptual objects with a modality because we associate their perceptible features with that modality. This has us going in a tight circle: A feature instance is associated with audition because it is instantiated in an auditory object, and a perceptual object is auditory because the features it instantiates are associated with audition. ${ }^{44}$ We will need something other than an appeal to the intuitive association of perceptual objects with a modality to get us a non-circular justification for associating inherited features with the inheriting modality. It can't just be that binding an initially visually supplied feature to an intuitively auditory object can, by itself, justify calling that feature auditory. After all, we haven't ruled out cross-modal objects - i.e., perceptual objects with features intuitively associated with different sensory modalities. We will need to dig beneath the phenomenal surface to understand why there is feature inheritance in the hope that this will provide a justification for associating the phenomenal feature with the inheriting modality. That is, we need to explain RR6's clause (i) in terms of the mechanisms responsible for feature inheritance. Barring this, we still have not fully satisfied constraint (4).

\footnotetext{
${ }^{44}$ This circularity worry is noted by Fulkerson with respect to his binding-based feature association condition (2014, p. 38). He attempts to block the worry by appeal, in part, to proper sensibles: Given an antecedent association of proper sensibles to modalities, we can type perceptual objects by the proper sensibles they contain and then group any other features that are bound to that object according the modality of the object. This might be sufficient for his purpose, which is to show that haptic touch is unisensory. However, it won't help our case: We are seeking a thorough going association rule that will tell us, among other things, which sensory modalities the proper sensibles belong to. But that is the very thing Fulkerson assumes.
} 
So what happens in one of these instances of inheritance? Conflicting determinates of the same determinable are pre-experientially attributed to (proto-)perceptual objects of different modalities. Perceptual objects, here, are bundles of bound features by which we encounter objective particulars. We can, for instance, be visually aware of some features of a thing and tactilely aware of others. The bundle of bound tactile features will be the tactile object, those of the visual features the visual object. ${ }^{45}$ (This doesn't require an ontologically robust thing that is the perceptual object.) If the resolution of this conflict yields a feature that could not have been supplied by one of the modalities being taken on as a feature of one of that modality's objects, then we have a proper instance of inheritance. (Otherwise, we have mere recalibration. ${ }^{46}$ )

But why should there be a conflict, given the fact that these are features of different perceptual objects? Because perceptual objects can be of the same objective particular (as in the look and feel of the cup in my hand) or of objects that are intimately related (as are sounds and their sources), such that having a determinate of a shared determinable in the one entails the same determinate in the other. We get conflicts when there is a clash of determinates in perceptual objects so related. ${ }^{47}$ To recognize such instances there has to be some pre-experiential mechanism linking objects and features across modalities and resolving apparent conflicts. This suggests that subexperiential information sharing across modalities underwrites inheritance.

If informational content is contributed to some mechanism and that mechanism uses that content in the determination of the phenomenal features of its perceptual objects, then we will have a justification for associating the phenomenal feature with the inheriting modality. This justification

\footnotetext{
${ }^{45}$ As noted above, this sort of intuitive classification of perceptual objects leads to a circularity worry, if it is to be used in associating perceptual features with sensory modalities. A better way of typing perceptual objects by modality is to do so in terms of the mechanisms that produce them (by binding features into those perceptual objects). Perceptual objects typed in this way can factor in a rule for associating features with modalities (see below).

${ }^{46}$ Notice that the basic mechanism for mere recalibration and inheritance is the same, which supports treating the McGurk and ventriloquism effects as broadly similar. Inheritance is a special case of recalibration.

${ }^{47}$ The sub-experiential recognition of this sort of relation between (proto-)perceptual objects is called the 'unity assumption' in the empirical literature. See Chen and Spence (2017) for a review.
} 
avoids the circularity worry while explaining the intuitive pull of the idea that, e.g., a feature of an auditory object ought to be associated with audition. It also gives us a better grip on why features aren't inherited arbitrarily: it is not adaptive to inherit features if there is no conflicting information to reconcile (e.g., no acceptable demarcation of the auditory mechanisms carries information regarding wavelength reflectance, so no conflict with vision can arise on this front). ${ }^{48}$ Notice, too, that the justification doesn't require that there be an instance of the relevant feature associated with the parent modality in the experience, only that the mechanism of the parent modality carry the sort of information that can be used to determine instances of the feature in the perceptual processing eventuating in the experience. This solves the problems from the ventriloquism effect in hemifield neglect patients and the persistence of recalibration once the parent mechanism is no longer receiving stimulation.

The key point, though, is that it is not phenomenology that is inherited, in the first instance, but information. ${ }^{49}$ Inheritance occurs sub-experientially, before any phenomenology arises. This leaves room for the modality to do some work in determining the phenomenal features, thereby justifying the claim that these features are properly associated with the modality. ${ }^{50}$ But even if informational content, alone, determines the feature instance, we can justify associating the feature with the

\footnotetext{
${ }^{48}$ Of course, it is possible that there are non-adaptive inheritance-like phenomena that we might want to capture under the label "inheritance". I have no strong leanings as to whether the resulting features should be associated with the "inheritor" mechanism. If so, then we can drop the shared determinable restriction in clause (b). Whether or not the feature satisfies the shared determinable restriction simply sorts the normal (adaptive) instances of inheritance from the odd ones.

${ }^{49} \mathrm{M}_{\mathrm{m}}$ 'inherits information' when it receives informational input from $\mathrm{M}_{\mathrm{k}}$ that (partially) determines an instance of $\mathrm{F}$, where no unimodal stimulation of $\mathrm{M}_{\mathrm{m}}$ would carry that information and where $\mathrm{M}_{\mathrm{k}}$ does carry that information in the sub-experiential processing resulting in E, either non-derivatively or through a chain of inheritance bottoming out in a mechanism that carries the information non-derivatively.

${ }^{50} \mathrm{On}$ this view the content might be associated with one (or more) modality, the resulting phenomenology with another. For example, we might continue to associate the localization content inherited, in the ventriloquism effect, with vision while associating the localization phenomenology with audition. This can accommodate a view like that of Bayne (2014) on which the contents of perceptual experiences involving sensory integration are multisensory while maintaining our guiding intuitions with respect to phenomenal features.
} 
inheritor modality due to its uptake of the information in the formation of its own perceptual objects.

Incorporating this into our association rule, we get:

(RR7) That instance of $F$ is associated with the modality individuated by $\mathrm{M}_{\mathrm{m}}$ if, and only if, there is some stimulus $R^{\prime}$ that is substantially similar to $R$ and, were just $M_{m}$ stimulated by $\mathrm{R}^{\prime}$, the resulting experience E' would either:

(a) have a corresponding instance of $\mathrm{F}$ or

(b) have a corresponding instance of a feature $F^{\prime}$ that is a determinate of the same determinable as $\mathrm{F}$, provided that

(i) $\mathrm{M}_{\mathrm{m}}$ inherits information from some other mechanism $\mathrm{M}_{\mathrm{k}}$ in the course of sub-experiential sensory processing (resulting in E) and

(ii) $\mathrm{M}_{\mathrm{m}}$ uses that information in determining the instance of $\mathrm{F}$ (in $\mathrm{E}$ ), as a feature of one of $\mathrm{M}_{\mathrm{m}}$ 's perceptual objects.

RR7 retains the advantages of RR6 while solving the circularity problem - it bases inheritance in the operation of the sensory mechanisms, forestalling the need to appeal to intuitions about the modality of perceptual objects/features in its application. However, in accepting this association rule, we lose something. Our previous versions of the rule make the sorting of phenomenal features empirically tractable - at least in principle - without requiring us to take on any theoretical commitments concerning the basis of the phenomenal features of perceptual experiences. We simply demarcate our mechanisms, isolate them, see what phenomenal features result from their stimulation, and compare with the phenomenal features of the original experience.

RR7, on the other hand, requires us to take a stand on what content a given mechanism has at various points in the course of processing a given stimulus and to endorse the supervenience of phenomenology on informational content. And once we have settled on a story that allows us to associate contents with mechanisms - and accepting the supervenience thesis - we will have to settle the further question concerning the point in the flow of information at which phenomenology emerges.

None of this is trivial, but until these issues are sorted RR7 will not rule on a large number of features. Furthermore, there is a problem lurking in RR7. To illustrate: Suppose Lexi takes a sip of root beer just as Gus says, "Joan is always gloomy in Los Angeles". RR7 will associate the root beer 
taste phenomenology with both taste and audition by clause (a) because unimodal sensory stimulation in either modality would have resulted in that phenomenology. But imagine two ways Lexi's synesthesia might operate: (1) When Lexi's synesthesia is triggered, processing of gustatory objects is stopped - the only taste-like phenomenology she has in those moments is due to her synesthesia (the mechanisms of which are part of her auditory mechanism). (2) When Lexi's gustatory system is stimulated, her synesthesia does not take effect. It seems clear that in (1) the root beer phenomenology should be attributed to audition and not to (the sense of) taste and in (2) it should be attributed to taste rather than audition. But, again, RR7 associates it with both regardless of how Lexi's synesthesia operates. And that means that clause (a) fails to satisfy constraint (4) just as clause (b) did in previous iterations of the rule. We have accepted that informational content plays a role in determining phenomenology to circumvent the problem for (b). Now we must do the same for (a):

(RR8) That instance of $F$ is associated with the modality individuated by $M_{m}$ if, and only if, there is some stimulus $R^{\prime}$ that is substantially similar to $R$ and, were just $M_{m}$ stimulated by $\mathrm{R}^{\prime}$, the resulting experience E' would either:

(a) have a corresponding instance of $\mathrm{F}$, where

(i) the instance of $\mathrm{F}$ in $\mathrm{E}$ was (partially) determined by information carried by $\mathrm{M}_{\mathrm{m}}$ or

(b) have a corresponding instance of a feature F' that is a determinate of the same determinable as $\mathrm{F}$, provided that

(i) $\mathrm{M}_{\mathrm{m}}$ inherits information from some other mechanism $\mathrm{M}_{\mathrm{k}}$ in the course of sub-experiential sensory processing (resulting in E) and

(ii) $\mathrm{M}_{\mathrm{m}}$ uses that information in determining the instance of $\mathrm{F}$ (in $\mathrm{E}$ ), as a feature of one of $\mathrm{M}_{\mathrm{m}}$ 's perceptual objects.

It will be helpful, in assessing RR8, to take a step back and remember why we are looking for an association rule in the first place. Primarily, we wanted an association rule to adjudicate the novel feature debate. We were interested in novel features because we were worried that theorizing about perceptual experience in a sense-by-sense manner would lead us to miss something crucial; namely, those features not explainable in terms of the individual (Aristotelian) senses. Now notice that, applying RR8 - and so using it to adjudicate the novel feature debate - requires a complete 
accounting of the flow of information through the sensory mechanisms and an account of when that information manifests consciously (both as representational content and phenomenal features). But, if we have this, we haven't left any features unexplained. It's just that the explanation doesn't appeal to the Aristotelian senses, it appeals to sensory mechanisms.

One might think that there is at least one theoretically interesting class of features in the vicinity of novel features that RR8 will reveal; namely, emergent features - i.e., informational content derived from multiple mechanisms, but not found in any of those mechanisms themselves, and phenomenal features supervening on this emergent information. Perhaps emergent features can be leveraged into a defense of novel features. Notice, though, that emergent information and features arise within as well as across the (acceptable demarcations of the) mechanisms of our pre-theoretical conceptions of the Aristotelian senses. For example, there is a chemoreceptor on the tongue that, when stimulated in isolation, contributes no phenomenal features. However, when it is stimulated along with gustatory receptors that $d o$ contribute phenomenal taste features, emergent features (called 'kokumi') result. These features include a strengthening of the basic tastes and features that are not entirely characterizable in terms of those basic tastes - e.g., 'heartiness' ${ }^{51}$ Kokumi receptors are part of the primary receptor of taste (i.e., they are chemoreceptors found on the tongue). And yet the result of a kokumi experience includes features that are more than the sum of the contributions of the sub-mechanisms giving rise to them - there is more to kokumi experience than one gets from the stimulation of any of the chemoreceptors of taste taken in isolation.

Given this, novel features will be a subset of emergent features. They are those emergent features that emerge from the interaction of sensory mechanisms we associate with distinct Aristotelian senses. If emergent features are going to form the basis of a defense of novel features,

\footnotetext{
${ }^{51}$ See Ohsu et al. (2010), Ueda et al. (1990); see also Brennan, Davies, Schepelmann, and Riccardi (2014), Maruyama, Yasuda, Kuroda, and Eto (2012), Yamamoto et al. (2020), Yang, Bai, Zeng, and Cui (2019). A plausible candidate for the emergent information on which the kokumi phenomenology supervenes is information pertaining to expected caloric content (Tang, Tan, Teo, and Forde 2020).
} 
the classification of some emergent features as novel will need to add explanatory power to our theory of perception. But the further classification of emergent features as novel will not add any explanatory power to our theory of perception: Once we are in a position to apply RR8, with its attendant account of emergent features, we have accounted for all the features arising from the sensory mechanisms (however demarcated from one another). And the senses, themselves, are to be individuated in terms of their mechanisms. So, the further classification of emergent features as novel is nothing more than an attempt to carve the results of RR8 to match pre-theoretical intuitions about the senses. (While intuition matching can be used to evaluate theoretical proposals, there is no theoretical value to intuition matching qua intuition matching.)

To illustrate, suppose that it were discovered that the putative novel feature involved in the experience of mintiness is a 'harmonizing' of the features provided by taste, smell, and trigeminal stimulation. And suppose, in addition, that, like kokumi, this feature only emerges when a further chemoreceptor is activated - one that does not produce any phenomenal features when stimulated in isolation. Furthermore, these (hypothetical) chemoreceptors are found midway between the gustatory receptors and the olfactory receptors - somewhere between the soft palate and the back of the nasal cavity - so that it is not entirely clear whether or not they belong to taste, smell, or neither. The flavor feature described here is certainly emergent. Whether or not it is novel depends on whether we attribute the hypothetical receptor to one of the Aristotelian senses. If, for example, we attribute the receptor to taste, then the feature will not be novel - it will be analogous to kokumi: the 'harmoniousness' will be associated with taste, though it 'harmonizes' features from multiple senses. If we don't attribute it to any Aristotelian sense, it will be novel. But choosing between these options doesn't have and further repercussions for our theorizing about mintiness. In particular, the classification of mintiness as novel, should we choose that option, does not help us explain anything further about the experience of mintiness. 
So the appeal to emergent features cannot be leveraged into a defense of novel features. But that was our last hope for finding a theoretical use for novel features. In short, RR8 renders the debate we were hoping to illuminate irrelevant. And so it fails to meet our third constraint, that the association rule make the novel feature debate substantive.

One might think this means we should look for a further tweak to the association rule that will fix these problems or for another way to formulate an association rule, other than our counterfactual strategy. ${ }^{52} \mathrm{I}$ am inclined to draw a different lesson: Multisensory experience (as construed in the novel feature debate) is a red herring - or, rather, a ladder to be kicked away. It was a useful step in philosophy of perception's recent march from a single-minded focus on vision through a broadened focus inclusive of other Aristotelian senses to the eventual recognition that these senses (intuitively construed) won't be useful in theorizing about perceptual experience. But having arrived at this point, we have no further use for novel features or multisensory accounts of perception.

Furthermore, we also wanted an association rule to determine the preferred individuation of the Aristotelian senses - at least for the novel feature debate. We just saw that none of our association rules met all four constraints. If this result for the novel feature debate generalizes, then there will be no good rule for determining the preferred demarcation of the mechanisms of the Aristotelian senses for the purposes of theorizing about the features of perceptual experiences.

\section{The problem generalizes}

In fact, the failure to find an adequate association rule for the novel feature debate does generalize to any debate that takes the pre-theoretical notions of the Aristotelian senses as a starting point for

\footnotetext{
${ }^{52}$ Regarding the counterfactual strategy, the only room left to maneuver concerns what it is to count as a result of the stimulation of a mechanism, but we've covered all the obvious ground there. Any further proposal will be both ad hoc and unlikely to help.
} 
theorizing about the contents and phenomenal character of perceptual experience. Any such debate needs an association rule that meets our four constraints (modifying the third constraint to apply to whichever debate is under consideration). But none of our association rules satisfied all four criteria. Perhaps, given that RR8 only ran afoul of the third constraint (to render the novel feature debate substantive), it will be useful for one of these other debates. If RR8 is going to be an acceptable association rule for one of these debates, then it will have to render that debate substantive. But RR8 clearly won't render any of these debates substantive because - as we've seen - all the explanatory work regarding the contents and phenomenal character of perception is already done by the time we are able to apply RR8.

Take the thick or thin contents debate, for example. The debate is over whether kind properties, such as being a pine tree, are ever contents of a perceptual (usually visual) experience. Insofar as the arguments in favor of thick contents rely on phenomenal contrast cases implicating a specific modality (e.g., Siegel 2006), we will need an association rule that tells us, for each kind property in the contents of a conscious experience, whether or not its correlated phenomenal character is associated with a sensory modality (such as vision) ${ }^{53} \mathrm{RR} 8$ will be able to do this, but once we are in a position to apply RR8 we will already know all there is to know about which demarcations of, e.g., the visual mechanism carry which contents and result in which phenomenal features. Those who favor thick contents will prefer a demarcation of the visual mechanism that associates the phenomenal features correlated with certain kind properties with vision. Those who favor a thin account will prefer a demarcation that doesn't. As with the novel feature debate, picking one demarcation over the other will have no further implications for any of our theorizing about perceptual content and phenomenology, though. All that theorizing will have already been done.

\footnotetext{
${ }^{53}$ One could, of course, pursue the thick/thin debate without adverting to specific sensory modalities. But this is just to concede the point that our pre-theoretical notions of the senses are not an adequate basis for theorizing about such matters.
} 
A final comment: I am not saying that there could be no theoretical use for our pre-theoretical notions of the senses, just that there will be none with respect to explaining perceptual content and phenomenology. Nor am I saying that we should abandon any differentiation of the sensory mechanisms and focus just on a single modality, perceiving, in our explanations of content and phenomenology (as Speaks (2015) suggests). ${ }^{54}$ My approach is more akin to the sensory pluralism of Macpherson (2011b, 2011c) and Fulkerson (2014), minus the extra step of declaring some demarcations of the sensory mechanisms to be versions of the Aristotelian senses. I omit the step because it adds nothing to the explanatory power of our perceptual theories.

\section{Conclusion}

I have argued that any association rule that will be acceptable for the purposes of informing the novel feature debate must meet four requirements: (1) It must answer 'yes' or 'no' to the question 'Is this feature associated with that modality?' for every feature and modality. (2) It must conform to our guiding intuitions regarding the correct associations of proper sensibles and the results of crossmodal recalibration effects. (3) It must render the debate substantive. (4) It must appeal to the stimulation states of sensory mechanisms.

Given the general form of such a rule - roughly, a feature of a perceptual experience belongs to a given modality if, and only if, it could/would result from the stimulation of that modality in a unimodal experience - alterations can be made along the dimensions of (a) the demarcation of the sensory mechanisms by which we individuate the sensory modalities, (b) the stimulation received by that mechanism, and (c) what counts as a result of the stimulation of that mechanism. It was found that no variation on these parameters can satisfy all the constraints for an adequate association rule. Without an association rule we have no way to adjudicate the novel feature debate. Furthermore, our

\footnotetext{
${ }^{54}$ Indeed, the results canvased here suggest that the relevant mechanisms won't divide into perceptual and conceptual any more neatly than they will divide into perceptual modalities.
} 
attempt to find an adequate association rule revealed that novel features are not an interesting theoretical notion.

The failure to find an adequate association rule generalizes, undermining any attempt to theorize about the contents and phenomenal character of perception in terms of the Aristotelian senses. It is time to slough off the remaining baggage of the sense-by-sense approach to theorizing about the features of perceptual experiences in favor of an approach that proceeds, not by appeal to under-defined notions of the sensory modalities, but in terms of the precisely demarcated sensory mechanisms. Doing so will be illuminating for a number of philosophical debates about perception. To cite a few that have received passing mention here: (1) Whether or not we need a Kantian intuition of space to have spatial perception, (2) the thick/thin contents of perceptual experience, and (3) a naturalistic account of the contents and phenomenology of perceptual experiences.

The discussion here has already suggested how we might proceed with this project. The first step is to account for the informational contents carried in the perceptual mechanisms and how that informational content relates to the representational contents of perception. A good bit of work has already been done in this area, though there is certainly more to accomplish (e.g., Neander 2017; Shea 2018). The second step is to explain the relationship between phenomenology and informational/representational content. If phenomenology is determined by content or by content + mechanism, then we will be able to account for all the features of perceptual experiences once we have completed the first step. Even if it isn't, there is certainly a close connection between the content and phenomenology of a perceptual experience, so we will have gone some way towards explaining phenomenology once we have sorted the issues concerning mechanisms and contents. Furthermore, by focusing on clearly demarcated mechanisms, we make the question of the phenomenology of an experience resulting from the operation of that mechanism empirically tractable - at least in principle. We only need the means of isolating that mechanism and testing its deliverances (in light of our theories of informational content, representation, and phenomenology). This is not something 
that our pre-theoretical notions of the senses afford, given the uncertainty about how precisely to demarcate their mechanisms.

Acknowledgments Thanks to James Van Cleve, Janet Levin, John Hawthorne, and participants in the European Philosophy of Language and Mind Network's fifth annual meeting for helpful comments on earlier drafts of this paper. Thanks also to two anonymous referees for this journal, whose comments led to many improvements. 


\section{Appendix}

Tables summarizing the association rules, how they fared with respect to the four constraints on an acceptable association rule, and - for those that failed to satisfy constraint 2 (regarding our guiding feature association intuitions), how they fared with respect to intuitions concerning proper sensibles, the McGurk effect, and the ventriloquism effect, are given below.

\begin{tabular}{|c|c|c|c|}
\hline & $\begin{array}{l}\text { demarcation of the } \\
\text { sensory mechanisms }\end{array}$ & $\begin{array}{l}\text { what is the relevant stimulation } \\
\text { for assessing the counterfactual? }\end{array}$ & $\begin{array}{l}\text { what counts as a result of stimulation of } \\
\text { that mechanism? }\end{array}$ \\
\hline OCR & indeterminate & equivalent to actual & what could result \\
\hline RR1 & determinate & equivalent to actual & what could result \\
\hline RR2 & determinate & equivalent to actual & what would result \\
\hline RR3 & determinate & substantially similar to actual & what would result \\
\hline RR4 & determinate & unconstrained & what would result \\
\hline RR5 & determinate & substantially similar to actual & $\begin{array}{l}\text { what would result but allowing differences } \\
\text { in determinate of same determinable }\end{array}$ \\
\hline RR6 & determinate & substantially similar to actual & $\begin{array}{l}\text { what would result but allowing restricted } \\
\text { feature inheritance from other mechanisms }\end{array}$ \\
\hline RR7 & determinate & substantially similar to actual & $\begin{array}{l}\text { what would result but allowing restricted } \\
\text { inheritance of information from other } \\
\text { mechanisms, with phenomenology } \\
\text { supervening on informational } \\
\text { content }+ \text { modality }\end{array}$ \\
\hline RR8 & determinate & substantially similar to actual & $\begin{array}{l}\text { what would result, provided what did } \\
\text { result was (partially determined by } \\
\text { information carried in the mechanism) but } \\
\text { allowing restricted inheritance of } \\
\text { information as in } R R 7\end{array}$ \\
\hline
\end{tabular}

Table 1. Summary of the association rules considered herein is given, with respect to the dimensions along which those rules varied.

\begin{tabular}{|c|c|c|c|c|}
\hline & $\begin{array}{l}\text { Rules on every feature for } \\
\text { each modality }\end{array}$ & $\begin{array}{l}\text { Satisfies guiding } \\
\text { intuitions }\end{array}$ & $\begin{array}{c}\text { Makes novel } \\
\text { feature debate } \\
\text { substantive }\end{array}$ & $\begin{array}{c}\text { Sufficiently grounded in } \\
\text { stimulation states of } \\
\text { mechanisms }\end{array}$ \\
\hline OCR & no & no & yes & no \\
\hline RR1 & yes & no & yes & yes \\
\hline RR2 & yes & no & yes & yes \\
\hline RR3 & yes & no & yes & yes \\
\hline
\end{tabular}




\begin{tabular}{|c|c|c|c|c|}
\hline & $\begin{array}{l}\text { Rules on every feature for } \\
\text { each modality }\end{array}$ & $\begin{array}{l}\text { Satisfies guiding } \\
\text { intuitions }\end{array}$ & $\begin{array}{l}\text { Makes novel } \\
\text { feature debate } \\
\text { substantive }\end{array}$ & $\begin{array}{c}\text { Sufficiently grounded in } \\
\text { stimulation states of } \\
\text { mechanisms }\end{array}$ \\
\hline RR4 & yes & no & yes & yes \\
\hline RR5 & yes & uncertain & yes & no \\
\hline RR6 & yes, but circular & yes, but circular & yes & no \\
\hline RR7 & $\begin{array}{c}\text { yes, pending theoretical } \\
\text { results }\end{array}$ & uncertain & no & no \\
\hline RR8 & yes & uncertain & no & yes \\
\hline
\end{tabular}

Table 2a. Summary of results for each association rule with respect to the four constraints on an acceptable association rule.

\begin{tabular}{|c|ccc|}
\hline \multicolumn{2}{|c}{ Proper Sensibles } & McGurk Effect & Ventriloquism Effect \\
\hline Intuition & non-novel, unique to a modality & auditory & auditory \\
\hline OCR & non-novel, not unique & auditory & auditory \\
RR1 & non-novel, not unique & auditory & auditory \\
RR2 & non-novel, unique & non-auditory & non-auditory \\
RR3 & non-novel, unique & auditory & non-auditory \\
RR4 & non-novel, unique & auditory & non-auditory \\
\hline
\end{tabular}

Table $2 \mathbf{b}$. A comparison of the guiding feature association intuitions for the relevant features in our test cases and the results for those association rules that were rejected for failing to satisfy this constraint. (These results apply to our generalized notion of an acceptable demarcation of the sensory mechanisms.) 


\section{References}

Auvray, M., \& Spence, C. (2008). The multisensory perception of flavor. Consciousness and Cognition 17, 1016-1031.

Bayne, T. (2014). The multisensory nature of perceptual consciousness. In D. J. Bennett \& C. S. Hill (Eds.), Sensory Integration and the Unity of Consciousness (pp. 15-36). Cambridge, MA: MIT Press.

Berger, C. C., \& Ehrsson, H. H. (2013). Mental imagery changes multisensory perception. Current Biology, 23, 1367-1372.

Bertelson, P., Vroomen, J., de Gelder, B., \& Driver, J. (2000). The ventriloquism effect does not depend on the direction of deliberate visual attention. Perception and Psychophysics, 62(2), 321-332.

Blauert, J. (1997). Spatial hearing, 2nd ed. Cambridge, MA: MIT Press.

Bourget, D. (2017). Representationalism and sensory modalities: an argument for intermodal representationalism. American Philosophical Quarterly, 54(3), 251-268.

Brennan, S. C., Davies, T. S., Schepelmann, M., \& Riccardi, D. (2014). Emerging roles of the extracellular calcium-sensing receptor in nutrient sensing: control of taste modulation and intestinal hormone secretion. British Journal of Nutrition, 111, S16-S22.

Briscoe, R. (2016). Multisensory processing and perceptual consciousness: part I. Philosophy Compass, 11(2), 121-133.

Briscoe, R. (2017). Multisensory processing and perceptual consciousness: part II. Philosophy Compass, 12, 1-13.

Briscoe, R. (2019). Bodily awareness and novel multisensory features. Synthese, https://doi.org/10.1007/s11229-019-02156-2

Caclin, A., Soto-Faraco, S., Kingstone, A., \& Spence, C. (2002) Tactile 'capture' of audition, Perception and Psychophysics, 64, 616-630.

Callan, A., Callan, D., \& Ando, H. (2015). An fMRI study of the ventriloquism effect. Cerebral Cortex, 25, 4248-4258

Chen, Y.-C., \& Spence, C. (2017). Assessing the role of the 'unity assumption' on multisensory integration: a review. Frontiers in Psychology, 8, 445. https://doi.org/10.3389/fpsyg.2017.00445

Coady, C. A. J. (1974). The senses of martians. Philosophical Review, 83(1), 107-125.

Connolly, K. (2014). Making sense of multiple senses. In R. Brown (Ed.), Consciousness inside and out: phenomenology, neuroscience, and the nature of experience (pp. 351-364). New York: Springer.

Crane, T. (2003). The intentional structure of consciousness. In Q. Smith \& A. Jokic (Eds.), Consciousness: new philosophical perspectives (pp. 33-56). Oxford: Oxford University Press.

Crane, T. (2007). Intentionalism. In A. Beckermann \& B. P. McLaughlin (Eds.), Oxford handbook to the philosophy of mind (pp. 365-373). Oxford: Oxford University Press.

Deroy, O., Spence, C., \& Noppeney, U. (2016). Causal metacognition: monitoring uncertainty about the causal structure of the world. Trends in Cognitive Sciences, 20, 736-747.

Dretske, F. (1981). Explaining behavior: reasons in a world of causes. Cambridge, MA: MIT Press.

Dretske, F. (1988). Knowledge and the flow of information. Cambridge, MA: MIT Press.

Dretske, F. (1995). Naturalizing the mind. Cambridge, MA: MIT Press.

Driver, J., \& Noesselt, T. (2008). Multisensory interplay reveals crossmodal influences on 'sensoryspecific' brain regions, neural responses, and judgments. Neuron, 57, 11-23.

Fulkerson, M. (2011). The unity of haptic touch. Philosophical Psychology, 24:4, 493-516. 
Fulkerson, M. (2014a). Explaining multisensory experience. In R. Brown (Ed.), Consciousness inside and out: phenomenology, neuroscience, and the nature of experience (pp. 365-373). New York: Springer.

Fulkerson, M. (2014b). The first sense: a philosophical study of human touch. Cambridge, MA: MIT Press.

Fulkerson, M. (2014c). Rethinking the senses and their interactions: the case for sensory pluralism. Frontiers in Psychology, 5, 1-14.

Ghazanfar, A., \& Schroeder, C. E. (2006). Is neocortex essentially multisensory? Trends in Cognitive Sciences, 10, 278-285.

Gray, R. (2013). Is there a space of sensory modalities? Erkenntnis, 78(6), 1259-1273.

Grice, H. P. (1962). Some remarks about the senses. In R. J. Butler (ed.), Analytic Philosophy, First Series (pp. 133-153). Oxford: Oxford University Press.

Heil, J. (1983). Perception and cognition. Berkeley, CA: University of California Press.

Keeley, B. (2002). Making sense of the senses: individuating modalities in humans and other animals. Journal of Philosophy, 99(1), 5-28.

Leo, F., Bolognini, N., Passamonti, C., Stein, B. E., \& Ladavas, E. (2008). Cross-modal localization in hemianopia: new insights on multisensory integration. Brain, 131, 855-865.

Lycan, W. G. (1996). Consciousness and experience. Cambridge, MA: MIT Press.

Macpherson, F. (2011a). Cross-modal experiences. Proceedings of the Aristotelian Society, 111(3), 429-468.

Macpherson, F. (2011b). Individuating the senses. In F. Macpherson (Ed.), The senses: classical and contemporary readings (pp. 3-36). Oxford: Oxford University Press.

Macpherson, F. (2011c). Taxonomizing the senses. Philosophical Studies, 153(1), 123-142.

Macpherson, F. (2014). The space of sensory modalities. In D. Stokes, M. Matthen, \& S. Biggs (Eds.), Perception and its modalities (pp. 434-463). Oxford: Oxford University Press.

Maruyama, Y., Yasuda, R., Kuroda, M., \& Eto, Y. (2012). Kokumi substances, enhancers of basic tastes, induce responses in calcium-sensing receptor expressing taste cells. PLoS ONE, 7(4), e34489, https://doi.10.1371/journal.pone.0034489

Matthen, M. (2015). Individuation of the senses. In M. Matthen (Ed.), The Oxford handbook of perception (pp. 567-586). Oxford: Oxford University Press.

Millikan, R. (1984). Language, thought and other biological categories. Cambridge, MA: MIT Press.

Neander, K. (2017). A mark of the mental. Cambridge, MA: MIT Press.

Nelkin, N. (1990). Categorising the senses. Mind and Language, 5, 149-165.

Nudds, M. (2004). The significance of the senses. Proceedings of the Aristotelian Society, 104(1), 31-51.

O'Callaghan, C. (2008). Seeing what you hear: cross-modal illusions and perception. Philosophical Issues, 18, 316-338.

O’Callaghan, C. (2012). Perception and multimodality. In E. Margolis, R. Samuels, \& S. Stich (Eds.), Oxford handbook of philosophy of cognitive science, (pp. 92-117). Oxford: Oxford University Press.

O’Callaghan, C. (2014a). Intermodal binding awareness. In D. J. Bennett \& C. S. Hill (Eds.), Sensory integration and the unity of consciousness (pp. 73-103). Cambridge, MA: MIT Press.

O'Callaghan, C. (2014b). Not all perceptual experience is modality specific. In D. Stokes, M. Matthen, \& S. Biggs (Eds.), Perception and its modalities (pp. 133-165). Oxford: Oxford University Press.

O'Callaghan, C. (2015). The multisensory character of perception. Journal of Philosophy, 112(10), 551-569.

O’Callaghan, C. (2017a). Beyond vision: philosophical essays. Oxford: Oxford University Press.

O'Callaghan, C. (2017b). Grades of multisensory awareness. Mind and Language, 32(2), 155-181. 
O'Callaghan, C. (2019). A multisensory philosophy of perception. Oxford: Oxford University Press.

O'Dea, J. (2006). Representationalism, supervenience, and the cross-modal problem. Philosophical Studies, 130(2), 285-295.

Ohsu, T., Amino, Y., Nagasaki, H., Yamanaka, T., Takeshita, S., Hatanaka, T., Maruyama, Y., Miyamura, N. \& Eto, Y. (2010). Involvement of the calcium-sensing receptor in human taste perception. The Journal of Biological Chemistry, 285(2), 1016-1022.

Papineau, D. (1984). Reality and representation. Oxford: Blackwell.

Richardson, L. (2014). Non sense-specific perception and the distinction between the senses. Res Philosophica, 91(2), 215-239.

Rock, I., \& Harris, C. S. (1967). Vision and touch. Scientific American, 216(5), 96-107.

Rock, I., \& Victor, J. (1964). Vision and touch: an experimentally created conflict between the two senses. Science, 143(3606), 594-596.

Roxbee-Cox, J. W. (1970). Distinguishing the senses. Mind, 79, 1530-1550.

Schroeder, C. E., \& Foxe, J. (2005). Multisensory contributions to low-level, 'unisensory' processing. Current Opinion in Neurobiology, 15, 454-458.

Shea, N. (2018). Representation in cognitive science. Oxford: Oxford University Press.

Shimojo, S., \& Shams, L. (2001). Sensory modalities are not separate modalities: plasticity and interactions. Current Opinion in Neurobiology, 11, 505-509.

Shinn-Cunningham, B. G., Santarelli, S., Kopco, N. (2000). Tori of confusion: binaural localization cues for sources within reach of a listener. Journal of the Acoustical Society of America, 107(3), 1627-1636.

Siegel, S. (2006). Which properties are represented in perception? In T. S. Gendler \& J. Hawthorne (Eds.), Perceptual experience (pp. 481-503). Oxford: Oxford University Press.

Soroker, N., Calamaro, N., \& Myslobodsky, M. (1995a). 'McGurk illusion' to bilateral administration of sensory stimuli in patients with hemispatial Neglect. Neuropsychologia, 33, 461-470.

Soroker, N., Calamaro, N., \& Myslobodsky, M. (1995b). Ventriloquism effect reinstates responsiveness to auditory stimuli in the 'ignored' space in patients with hemispatial neglect. Journal of Clinical and Experimental Neuropsychology, 17, 243-255.

Speaks, J. (2015). The phenomenal and the representational. Oxford: Oxford University Press.

Spence, C. (2015a). Cross-modal perceptual organization. In J. Wagemans (Ed.), The Oxford Handbook of Perceptual Organization (pp. 649-664). Oxford: Oxford University Press.

Spence, C. (2015b). Multisensory flavor perception. Cell, 161, 24-35.

Spence, C. (2016). Oral referral: on the mislocalization of odours to the mouth. Food Quality and Preference, 50, 117-128.

Spence, C., \& Bayne, T. (2014). Is consciousness multisensory? In D. Stokes, M. Matthen, S. Biggs (Eds.), Perception and its modalities (pp. 95-131). Oxford: Oxford University Press.

Spence, C., \& Frings, C. (2020). Multisensory feature integration in (and out) of the focus of spatial attention. Attention, Perception, and Psychophysics, 82(1), 363-376.

Strawson, P. F. (1959). Individuals. London: Methuen Press.

Stevenson, R. J. (2009). The psychology of flavor. Oxford: Oxford University Press.

Tang, C. S., Tan, V. W. K., Teo, P. S., Gorde, C. G. (2020). Savoury and kokumi enhancement increases perceived calories and expectation of fullness in equicaloric beef broths. Food Quality and Preference, 83, 103897.

Ueda, Y., Sakaguchi, M., Hirayama, K., Miyajima, R., \& Kimizuka, A. (1990). Characteristic flavor constituents in water extract of garlic. Agricultural and Biological Chemistry, 54(1), 163 169.

Vignemont, F. de (2014). A multimodal conception of bodily awareness. Mind, 123(492), 989-1020. 
Yamamoto, M., Terada, Y., Motoyama, T., Shibata, M., Saito, T., \& Ito, K. (2020). N-terminal $[\mathrm{Glu}]_{3}$ moiety of $\gamma$-glutamyl peptides contributes largely to the activation of human calciumsensing receptor, a kokumi receptor. Bioscience, Biotechnology, and Biochemistry, https://doi.org/10.1080/09168451.2020.1743169

Yang, J., Bai, W., Zeng, Z., and Cui, C. (2019). Gamma glutamyl peptide: the food source, enzymatic synthesis, kokumi-active and the potential functional properties - a review. Trends in Food Science \& Technology, 91, 339-346. 